\title{
High-throughput fluorescence detection using an integrated zone-plate array $\dagger$
}

\author{
Ethan Schonbrun, ${ }^{* a}$ Adam R. Abate, ${ }^{b}$ Paul E. Steinvurzel, ${ }^{a}$ David. A. Weitz ${ }^{a b}$ and Kenneth B. Crozier ${ }^{a}$ \\ Received 10th November 2009, Accepted 16th December 2009 \\ First published as an Advance Article on the web 18th January 2010 \\ DOI: 10.1039/b923554j
}

\begin{abstract}
Microfluidic devices enable massive parallelization of sample manipulation and delivery, but a similarly parallelized and integrated optical detection system does not yet exist. Standard large numerical aperture wide field or scanning optical systems are not capable of the large field of view and detection sensitivity required to collect fluorescence from parallel arrays of microfluidic devices. Instead, we present a fluorescence measurement platform based on a microfabricated zone-plate array integrated into a parallelized microfluidic device. The zone-plate array is orientated so that a single high numerical aperture zone plate is aligned to read out the fluorescence from each of 64 output channels of a dropmaking device. The parallelization of microfluidics and optics produces an integrated system capable of analysis of nearly 200000 drops per second.
\end{abstract}

\section{Introduction}

Optical detection in biological assays is frequently performed by quantification of a fluorescence signal in a discrete volume or container. In order to screen a larger number of samples in a shorter time, two phase microfluidic technology has recently demonstrated the ability to produce picolitre sized containers of fluid, called drops, at rates of several kilohertz per device. ${ }^{1,2}$ Microfluidic devices can be parallelized on a single chip using lithographic techniques, ${ }^{3}$ further increasing the rate at which samples can be produced and manipulated. Consequently, droplet based microfluidics is ideally suited for carrying out highthroughput screening and other applications that require a large number of reactions to be executed. However, as the size of devices scale up, optical detection becomes increasingly difficult because of the tradeoff between field of view and collection efficiency. In optical imaging systems requiring a wide field of view and a small form factor, the use of a lens array instead of a single lens can avoid this tradeoff..$^{4-6}$ Here, we demonstrate a zone-plate array optical detection system that is integrated into a massively parallel microfluidic device. The zone-plate array efficiently collects fluorescence signals at well defined regions spanning a large area. Consequently, this multilayer microfluidic and optical detection platform enables parallelization not possible using a single microscope objective lens.

\section{Microfluidic/micro-optic design}

The high-throughput microfluidic device presented in this paper produces drops traveling through 64 parallel microfluidic channels. The drops are formed in a single flow-focus drop maker and then are subsequently split by six layers of drop splitters, shown in Fig. 1(a). Each channel is $15 \mu \mathrm{m}$ wide ( $\left.w_{\text {channel }}\right), 12 \mu \mathrm{m}$ tall, and

${ }^{a}$ School of Engineering and Applied Sciences, Harvard University, Cambridge, USA. E-mail: schonbru@seas.harvard.edu

${ }^{b}$ Department of Physics, Harvard University, Cambridge, USA

$\uparrow$ Electronic supplementary information (ESI) available: Movie showing simultaneous fluorescence detection of 64 channels producing approximately 30000 drops per second, played back at $1 / 50$ of real time. See DOI: $10.1039 / \mathrm{b} 923554 \mathrm{j}$ the channel spacing is $30 \mu \mathrm{m}$, shown in Fig. 1(b). The drops are plug-like, conforming to the channel width and height, and are approximately $25 \mu \mathrm{m}$ long. The drop volume is therefore $\sim 4 \mathrm{pL}$. This parallel sample-delivery system produces a large $4 \mathrm{~mm}^{2}$ region of quickly moving drops that require high-speed and sensitive optical measurement. The image in Fig. 1(b), for example, was taken with a low magnification objective lens $(10 \times)$, but the field of view is not sufficient for imaging the entire device. It would be possible to use an objective with an even lower magnification to increase the field of view, but such an
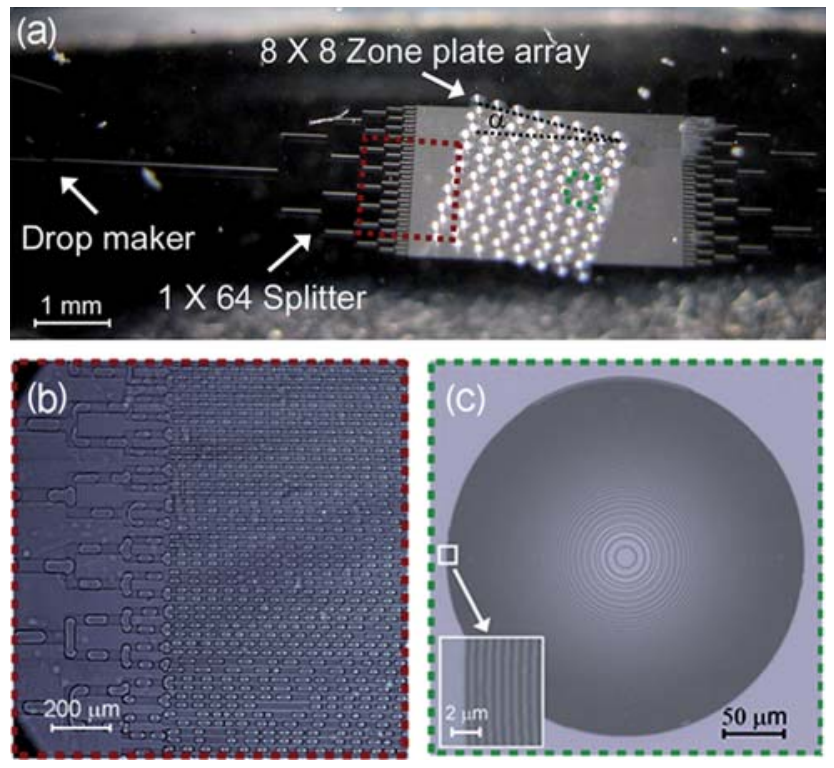

Fig. 1 Integration of parallel microfluidics and optics. (a) Microscope image of the microfluidic and optical device, consisting of a flow-focus drop maker, six layers of drop splitters, followed by 64 parallel microfluidic channels, and a zone-plate array which is aligned to the microfluidic channel array at a tilt angle, $\alpha$. (b) Zoom-in of region indicated by dashed crimson square in (a), showing the last three layers of the dropsplitter microfluidic device. Image is obtained with a $10 \times, 0.3 \mathrm{NA}$ objective lens. (c) Zoom-in of region indicated by dashed green square in (a), showing a single PDMS molded zone plate. 
objective would likely have an even smaller numerical aperture (NA) and consequently lower light collection efficiency than that of the lens used in Fig. 1(b) (NA 0.3).

Instead of using a single low NA objective, our optical detection system consists of an array of high NA zone plates, each confocally detecting from a localized region in a unique channel. Increasing the NA is extremely important because collection efficiency scales approximately with the square of NA. Using the zone-plate array, the fluorescence in every microfluidic channel is measured simultaneously without scanning, in contrast to what is frequently done in parallel detection systems. ${ }^{7}$ The lens array is illuminated by a fluorescence excitation laser and is imaged onto a high-speed CMOS camera using a unity magnification 0.13 NA telescope, shown in Fig. 2. Zone plates are planar in contrast to lenses that use refraction across curved surfaces. Consequently, they can easily be integrated into flat substrates in multilayer designs. Both the microfluidic device and the zone plates are fabricated from PDMS using soft lithography. In addition to the advantages of being planar, zone plates are ideal in lens arrays because their focal length can be defined with much higher accuracy and precision than refractive lens arrays. ${ }^{8}$ Zone plates can obtain extremely large $\mathrm{NAs}^{9-11}$ and can be matched to the focusing medium, consequently minimizing spherical aberration.

Each zone plate performs two functions. The first is to produce a focused excitation spot inside each microfluidic channel in the array. The second is to collect the fluorescence emission over each detection region efficiently. The zone plates are designed to focus light through a $170 \mu \mathrm{m}\left(t_{\text {cover }}\right)$ thick No. 1 cover slip that is bonded to the top surface of the microfluidic device, as shown in Fig. 3(a). Zone plates have substantial chromatic aberration, and consequently the focal length at the fluorescence excitation wavelength of $532 \mathrm{~nm}$ is different from that at the fluorescence emission wavelength of $575 \mathrm{~nm}$. Chromatic aberration is not a problem, however, because the fluorescence excitation does not need to be focused into a diffraction limited spot, and instead should approximately match the width of the microfluidic channel, $w_{\text {channel. }}$. We design the zone plates to have a focal length of $180 \mu \mathrm{m}$ at a wavelength of $550 \mathrm{~nm}$, which is in between the

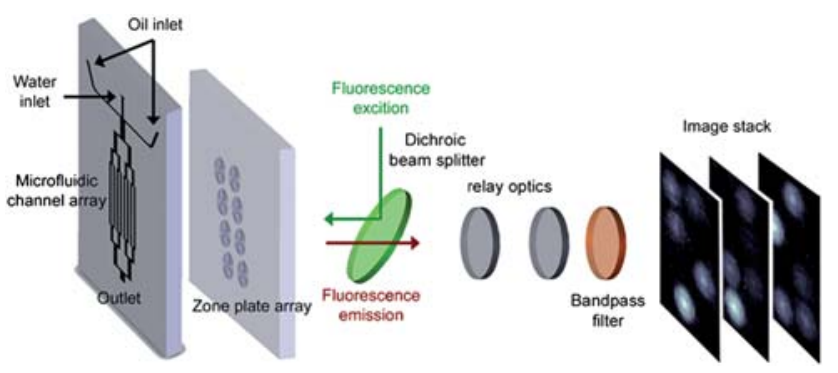

Fig. 2 Schematic of tilted zone-plate array detection system. The device has two layers, one containing the microfluidic channel array, and one containing the zone-plate array. Fluorescence excitation is introduced using a dichroic beam splitter and illuminates the zone-plate array. Fluorescence generated in the microfluidic device is collected by the zoneplate array and imaged onto a fast CMOS camera with unity magnification by two relay lenses. An image stack is stored in the camera that represents the simultaneous observation of each channel in the array. In this schematic, 8 total channels are illustrated, although the actual device has 64 channels and uses an $8 \times 8$ zone-plate array.
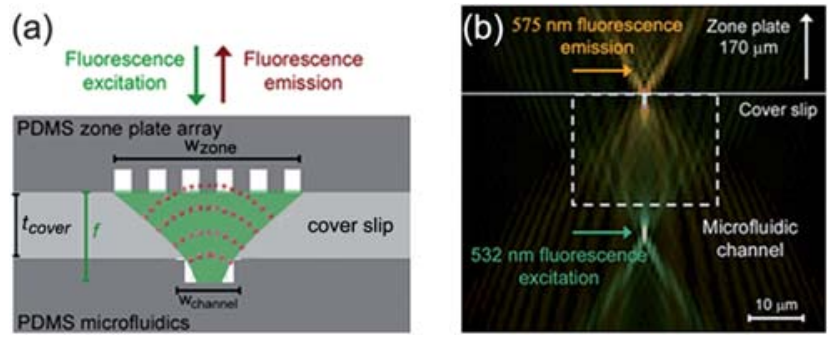

Fig. 3 (a) Schematic of cross-section of device. The two PDMS components, microfluidics and optics, sandwich a cover slip. The zoneplate width $\left(w_{\text {zone }}\right)$ is 15 times larger than the channel width ( $\left.w_{\text {channel }}\right)$ enabling large concentration of the excitation intensity and efficient collection of the emitted fluorescence. (b) Numerical simulation of the intensity distribution in the microfluidic channel for a case where the zone plate is illuminated with light at both the fluorescence excitation and emission wavelengths.

excitation and emission wavelengths. This is illustrated in Fig. 3(b), which shows the numerically simulated intensity distribution at the fluorescence excitation and emission wavelengths. The excitation light is focused beyond the channel depth, and therefore can have a spatial width that matches the channel size even though the diffraction limited focal spot size is much smaller.

The zone plates are solid immersion lenses because they are designed to focus through the glass cover slip. Solid immersion lenses have an NA that is enhanced by the refractive index of the background material, in this case glass. The width of each zone plate, $w_{\text {zone }}$, is $230 \mu \mathrm{m}$ resulting in an enhanced NA of 0.81 . The inner and outer radii of the concentric rings of the zone plate are given by $r_{n}=\sqrt{n \lambda f+n^{2} \lambda^{2} / 4}$, where $n$ is an integer, $f$ is the focal length, and $\lambda$ is the optical wavelength in glass. Due to refraction at the glass-water interface, the collected cone angle in the detection region is larger than that possible in the absence of the cover slip but with the same zone-plate diameter and focal length. In glass, each lens collects light over a half cone angle of $31^{\circ}$, corresponding to a half cone angle of $36^{\circ}$ in water. Assuming that the fluorescence is emitted isotropically in water, the collection efficiency of the zone plate is $C_{\mathrm{ZP}}=\xi / 2\left(1-\cos \theta_{\text {cone }}\right)=2.7 \%$, where $C_{\mathrm{ZP}}$ is the zone-plate collection efficiency, $\xi$ is the diffraction efficiency of $29 \%$, and $\theta_{\text {cone }}$ is the cone angle in water. This collection efficiency is equivalent to that of a $0.48 \mathrm{NA}$ objective lens with a transmission efficiency of 0.80 .

In addition to a high collection efficiency, each zone plate confocally filters the emitted fluorescence, ${ }^{12}$ meaning that the collection regions are well defined and do not overlap. Lower NA lens arrays have an overlapping field of view, which in this application would produce cross-talk from neighboring channels. The detection region of each zone plate is confined because of vignetting in the relay optics. The condition for vignetting is $\Delta x=r_{\text {relax }} f_{Z \mathrm{P}} / f_{\text {relay }}$, where $r_{\text {relay }}$ is the relay lens radius of $10 \mathrm{~mm}$, $f_{\text {relay }}$ is the relay lens focal length of $100 \mathrm{~mm}$, and $f_{\mathrm{ZP}}$ is the zoneplate focal length of $180 \mu \mathrm{m}$. Vignetting starts to occur in this system at a transverse distance of $9 \mu \mathrm{m}$ from the optical axis of the zone plate, so we expect an approximate detection region that has a width of $18 \mu \mathrm{m}$. 


\section{Excitation and collection characterization}

By aligning a single zone plate to a dye filled microfluidic channel, we can separately measure the enhancement due to increased excitation intensity and that due to increased collection efficiency, relative to the low NA telescope. To characterize the excitation, we focus the fluorescence excitation laser into the dye filled microfluidic channel using the zone plate. The emitted fluorescence is imaged onto a CCD camera using a microscope objective lens, shown in Fig. 4(a). The fluorescence, and consequently the excitation spot, has a full width at half maximum (FWHM) of $16 \mu \mathrm{m}$, which slightly overfills the channel width of $15 \mu \mathrm{m}$. Fig. 4(b) shows a one-dimensional projection of the image along the direction of flow with the background normalized to unity. The peak value is 19 times higher than background, meaning that the zone plate enhances the excitation efficiency by 19 times in comparison to the loosely focused incident beam.

The size and the relative enhancement of the zone-plate fluorescence collection are characterized by scanning a tightly focused fluorescence excitation spot, generated by a microscope objective lens, and quantifying the fluorescence captured by a zone plate and imaged onto a CCD camera. Fig. 5(a-c) show images of the zone plate through the relay optics when the excitation spot is 20,10 , and $0 \mu \mathrm{m}$ from the zone-plate center. Only fluorescence emitted from inside the detection region is collimated and captured by the relay optics, otherwise it is filtered out. Fig. 5(d) shows the integrated fluorescence over the aperture of the zone plate as a function of the excitation-spot position. The FWHM of the detection region of the zone-plate
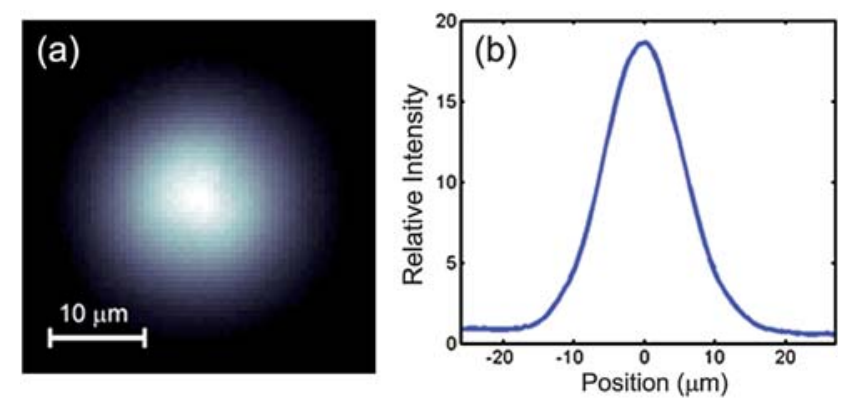

Fig. 4 (a) Experimentally measured zone-plate focal spot distribution. (b) One-dimensional projection of the focal spot distribution revealing a FWHM of $16 \mu \mathrm{m}$, slightly overfilling the channel width of $15 \mu \mathrm{m}$. imaging system is $21 \mu \mathrm{m}$, in reasonable agreement with that predicted by ray optics of $18 \mu \mathrm{m}$. Fluorescence emission from neighboring microfluidic channels will not be imaged onto the camera due to the fact that the detection region is smaller than the channel spacing. In addition, the detection region is also slightly larger than the expected drop size, so little of the fluorescence emission from each drop should be filtered out. Fig. 5(d) shows that the intensity of the fluorescence collected by the zone plate is 12 times that collected by the relay optics alone. The enhancement of a factor of 12 agrees well with the theoretical collection based on the NA and diffraction efficiency of the zone plate. The combination of the excitation and collection enhancement gives a total fluorescence signal enhancement of a factor of 230 inside the detection region.

\section{High-throughput fluorescence detection}

After the zone-plate characterization, we explore the performance of the entire array for collecting fluorescence from highspeed drops. An $8 \times 8$ zone-plate array is aligned to a 64 channel microfluidic device using a mask aligner and adhered to the opposite cover slip surface with a reversible bonding step. In this way, a single PDMS lens array can be peeled off and reused with several microfluidic devices. By tilting the two-dimensional zoneplate array by an angle $\alpha$ with respect to the one-dimensional microfluidic channel array, a single zone plate can be placed in each channel even though the zone-plate pitch is much larger than the channel spacing. A similar alignment has been performed with a one-dimensional pinhole array in the optofluidic microscope. ${ }^{13}$ By tilting a square zone-plate array by a small angle $(\alpha)$ with respect to the one-dimensional microfluidic channel array, each zone plate can be aligned into a unique microfluidic channel. The condition for this alignment is $\alpha=$ $\arctan (1 / n)$, where $n$ is the size of the square array, in this case 8 , which results in $\alpha=7.13^{\circ}$. For a microfluidic channel spacing $(a)$ of $30 \mu \mathrm{m}$, the required zone-plate pitch $(p)$ in the array is $p=a$ l $\sin (\alpha)=241 \mu \mathrm{m}$. Because $p$ is significantly larger than the microfluidic channel spacing, each zone plate can be much larger than the channel width and consequently collect more excitation energy with which to focus into the channel. To form drops, water containing $2 \mathrm{mM}$ resorufin dye and hydrocarbon oil (HFE-7500) with a surfactant of carboxylic acid of Krytox 157
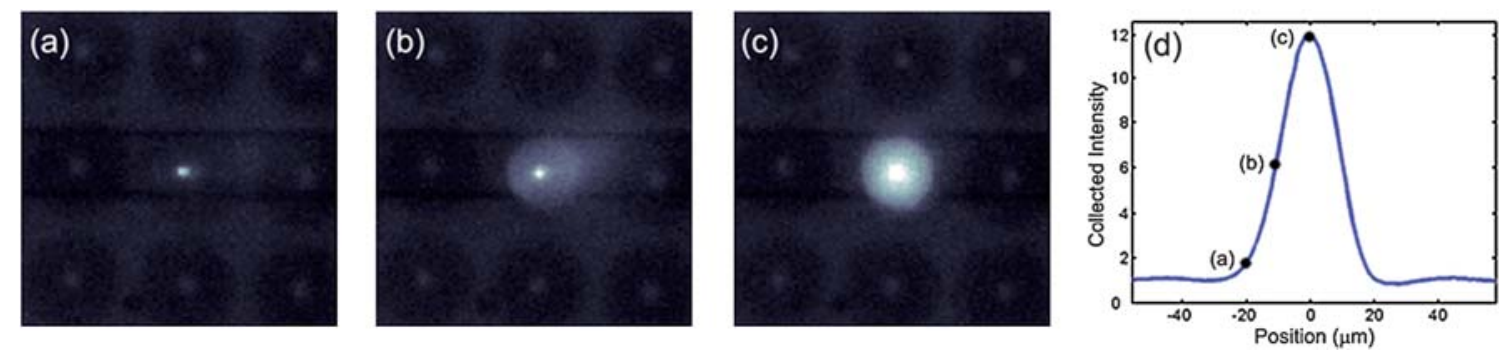

Fig. 5 Zone-plate collection efficiency and detection region. (a-c) A fluorescence excitation point source is scanned over the optical axis of a zone plate that is aligned to a dye filled microfluidic channel. The point source is located at a distance of $-20,-10$, and $0 \mu \mathrm{m}$ from the optical axis in (a), (b), and (c) respectively. Due to vignetting in the relay optics, the zone plate only collects fluorescence from a confined region close to the optical axis. When the fluorescence originates inside this confined region as it does for (c), it is collimated and sent towards the detector, and the zone plate appears bright. (d) Integrated fluorescence over the zone-plate aperture as a function of the location of the point source relative to the zone plate optical axis. 

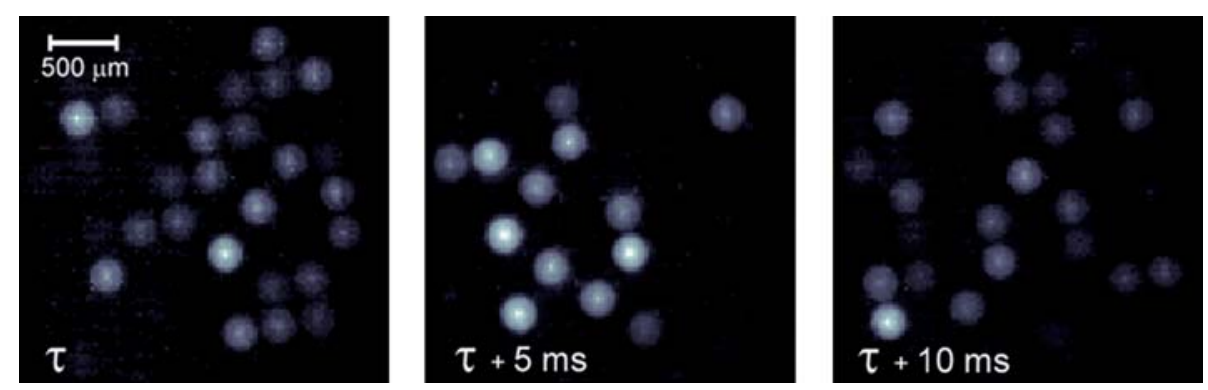

Fig. 6 Frames of the collected image stack. The three frames shown are captured at $5 \mathrm{~ms}$ increments. Each zone plate observes fluorescence in drops from a different microfluidic channel. In these images, drops are produced at $200 \mathrm{~Hz}$ in each channel. (Supplementary video $1 \uparrow$ shows a complete video of the image stack).

FSL at $1.8 \%$ by weight is loaded into the device as the inner and outer fluid phases respectively.

Fig. 6 shows three movie frames (ESI $\uparrow$ ) captured at $5 \mathrm{~ms}$ time intervals. The inner and outer flow rates of the drop maker are 8 and $20 \mu \mathrm{L} \mathrm{min}{ }^{-1}$, respectively. This results in drops being produced at approximately $200 \mathrm{~Hz}$. When a drop containing the fluorophore passes through the detection region of a zone plate, the collected fluorescence peaks due to the excitation and collection enhancement. The images in Fig. 6 are taken with a CMOS camera operating at $1 \mathrm{k}$ frames per second (fps). Using similar flow rates, we have also imaged the zone-plate array onto an EMCCD which has much greater sensitivity and lower pixel noise, but a maximum frame rate of approximately $300 \mathrm{fps}$. Drops with resorufin concentrations as low as $1 \mu \mathrm{M}$ have been imaged with signal to noise ratios of 20 , but only at rates approaching 100 drops per second per channel.

In order to obtain high-throughput detection, we utilize the high frame rates achievable with high-speed CMOS cameras. The zone-plate array is illuminated with $200 \mathrm{~mW}$ of fluorescence excitation light and a beam diameter that matches the diagonal length of the square zone-plate array, resulting in approximately $1.44 \mathrm{~mW}$ of excitation power incident on each zone plate. The CMOS camera is operated in sub-array mode where each frame is $128 \times 128$ pixels and each pixel is $22 \mu \mathrm{m}$ square. Because of the unity magnification telescope, the sub-array dimensions correspond to a $2.8 \mathrm{~mm}$ square field size, which is slightly larger than the $2 \mathrm{~mm}$ square zone-plate array. From the captured image stack, the fluorescence signal corresponding to each detection region is found by integrating the $\sim 100$ pixels associated with each zone plate. This is carried out for 62 of the 64 zone plates. The remaining two zone plates are misaligned, and do not produce appreciable signals. These appear dark in the image of Fig. 7(a), which is obtained by integrating the CMOS camera output over $1 \mathrm{~s}$. A Fourier transform of the time signals reveals that the drop rate in each channel is $2975 \mathrm{~Hz}$ when the drop maker is operated with inner and outer flow rates of 120 and $300 \mu \mathrm{L}$ $\mathrm{min}^{-1}$, respectively. When all 64 output channels are considered, this corresponds to an aggregate production of 190000 drops per second. Of these, 184000 drops per second are detected by the 62 operating zone plates. Fig. 7(b-e) show example fluorescence signal traces of four representative microfluidic channels when the CMOS camera is operating at $16000 \mathrm{fps}$. Assuming that the drops travel at the net volumetric flow rate, the average drop velocity is $615 \mathrm{~mm} \mathrm{~s}^{-1}$. In the $62.5 \mu \mathrm{s}$ in between captured frames of the image

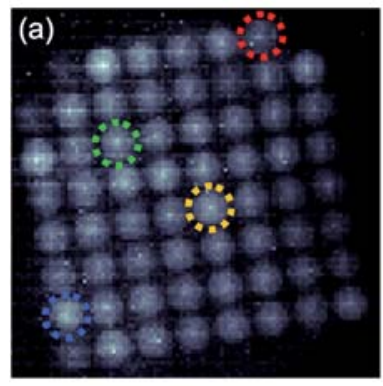

(b)

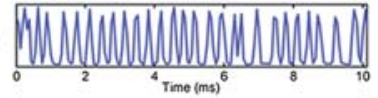

(c)

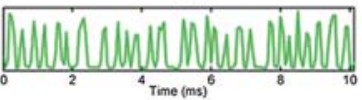

(d)

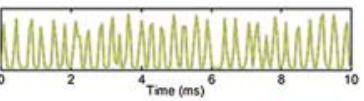

(e)

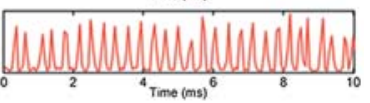

Fig. 7 Maximum detection throughput of 184000 drops per second. (a) CMOS camera image of drop fluorescence collected by zone-plate array, obtained by integrating output in time. Image illustrates simultaneous operation of 62 zone plates actively monitoring drops in 62 microfluidic channels. (b-e) Fluorescence captured by each zone plate can be plotted as a function of time, as shown for four arbitrary channels outlined by the blue, green, yellow, and red dotted lines. Drops are produced at $2975 \mathrm{~Hz}$ in each channel and frames are captured at 16000 per second.

stack, drops travel $38 \mu \mathrm{m}$. This, in combination with the excitation and collection volumes of each zone plate, sets the spatial resolution in the flow direction.

\section{Conclusions}

In this paper, we demonstrate a system capable of measuring the fluorescence from 184000 drops per second. This detection rate is several times faster than current flow cytometry systems, which traditionally have maximum rates of tens of thousands of measurements per second. ${ }^{14}$ This is achieved through the use of multiple sampling points in a $2 \mathrm{D}$ array format suitable for read out with a CMOS camera. It is interesting to consider the maximum detection throughput conceivable using current CMOS cameras. Fast CMOS cameras are capable of pixel throughputs of several billion per second when accounting for all the pixels in a three-dimensional image stack. The highest detection rate of resolvable quantities is dictated by Nyquist sampling. This reduces the total number by a factor of eight, when the two spatial dimensions and time are taken into account, which gives a maximum detection rate of several hundred million resolvable measurements per second. By parallelizing the sample delivery and measurement, the detection rate can theoretically approach the Nyquist sampling limit of the pixel throughput if 
the signal strength is sufficient for the required dynamic range. The device we demonstrate opens the possibility for achieving such detection rates through the use of large zone-plate arrays that span the spatial extent of the image sensor array. The results presented here are obtained with the CMOS camera operating at 260 million pixels per second. This is one order of magnitude below the maximum pixel throughput, and we therefore anticipate that even faster detection rates are possible with the technology we present.

We have demonstrated a novel platform for performing fluorescence measurements in microfluidic systems. By parallelizing both the optical detection and microfluidic drop formation, we have shown that this platform is particularly powerful for highthroughput detection. Using the enormous design flexibility of diffractive optics, on chip optical systems can be designed around microfluidic sample-delivery systems that considerably outperform standard off-chip optical systems. The flexibility in design of both sample-delivery systems and optical detection systems will enable high-throughput measurements to follow the pace of high-speed electronics.

\section{References}

1 G. M. Whitesides, Nature, 2006, 442, 368-373.

2 S. L. Anna, N. Bontoux and H. A. Stone, Appl. Phys. Lett., 2003, 82, 364-366.

3 D. R. Link, S. L. Anna, D. A. Weitz and H. A. Stone, Phys. Rev. Lett., 2004, 92, 054503.

4 A. W. Lohmann, Appl. Opt., 1989, 28, 4996-4998.

5 R. Völkel, M. Eisner and K. J. Weible, Microelectron. Eng., 2003, 6768, 461-472.

6 L. P. Lee and R. Szema, Science, 2005, 310, 1148-1150.

7 J. F. Dishinger and R. T. Kennedy, Electrophoresis, 2008, 29, 32963305.

8 H. J. Tiziani, R. Achi, R. N. Kramers and L. Wiegers, Appl. Opt., 1996, 35, 120-125.

9 D. Gil, R. Menon and H. I. Smith, J. Vac. Sci. Technol., B, 2003, 21, 2956-2960.

10 R. Brunner, M. Burkhardt, A. Pesch, O. Sandfuchs, M. Ferstl, S. Hohng and J. O. White, J. Opt. Soc. Am. A, 2004, 21, 1186-1191.

11 E. Schonbrun and K. B. Crozier, Appl. Phys. Lett., 2008, 92, 071112.

12 E. Schonbrun, W. N. Ye and K. B. Crozier, Opt. Lett., 2009, 34, $2228-$ 2230.

13 X. Cui, L. M. Lee, X. Heng, W. Zhong, P. W. Sternberg, D. Psaltis and C. Yang, Proc. Natl. Acad. Sci. U. S. A., 2008, 105, 10670-10675.

14 L. A. Sklar, Flow Cytometry for Biotechnology, Oxford University Press, New York, 2005. 